\title{
Continuing aspirin desensitization therapy in a patient with aspirin exacerbated respiratory disease needing sinus
} surgery

\author{
Nishioka GJ* \\ Willamette Ear Nose and Throat and Facial Plastic Surgery, Salem, Oregon, USA
}

\begin{abstract}
Triad asthma or Samter's triad, is more commonly referred to today as aspirin exacerbated respiratory disease (AERD). These patients suffer from three conditions: asthma, aspirin sensitivity, and nasal polyps (generally with paranasal sinus disease). The majority if not all of these patients require endoscopic sinus surgery (ESS) with nasal polypectomy as these patients often have the severest burden of sinus disease which is refractory to medical therapy. Discontinuing medication such as aspirin and non-steroidal anti-inflammatory (NSAID) medication is a universal tenet for elective surgery such as endoscopic sinus surgery (ESS) to prevent excessive bleeding. The interplay of these variables has created challenging management issues.

Aspirin desensitization therapy (ADT) in patients with AERD can achieve significant protection from asthma exacerbations associated with ingestion of aspirin, NSAIDs, and food items with salicylates. Because these patients generally need ESS, ADT is performed after ESS. As the prevalence of ADT increases situations will be encountered where a AERD patient is referred for nasal and sinus symptoms who is on ADT maintenance only to find they are in need of primary or revision ESS. This creates a dilemma for the surgeon regarding stopping or continuing ADT maintenance therapy which could require the patient to endure another ADT protocol which can have significant morbidity with the possibility of failure.

As the prevalence of ADT increases and the above scenarios become more commonplace this case report provides needed anecdotal data when the decision is made to continue ADT maintenance therapy in AERD patients who need primary or revision ESS. Otolaryngologists who encounter similar situations such as this one can use this published case to support their clinical decision making.
\end{abstract}

\section{Introduction}

Discontinuing medication such as aspirin and non-steroidal antiinflammatory (NSAID) medication is a universal tenet for elective surgery such as endoscopic sinus surgery (ESS) to prevent excessive bleeding. Patients with triad asthma or Samter's triad, more commonly referred today as aspirin exacerbated respiratory risease (AERD), suffer from three conditions: asthma, aspirin sensitivity, and nasal polyps. These patients are hypersensitive to aspirin (ASA), NSAIDs, and salicylate compounds in foods, and with ingestion develop an acute asthma exacerbation. Unfortunately, these patients often have the severest presentation and burden of sinus disease with pansinusitis and nasal polyposis refractory to non-surgical medical therapy.

In patients with AERD, aspirin desensitization therapy (ADT) provides protection from asthma exacerbations associated with ingestion of ASA, NSAIDs, and hidden salicylate compounds in food $[1,2]$. Because the majority if not all of these patients require ESS the desensitization procedure is performed after ESS because of the risk of severe bleeding associated with ASA and NSAIDs [3,4]. Occasionally, a situation is encountered where a AERD patient is referred for nasal and sinus symptoms who is on aspirin desensitization maintenance therapy only to find they are in need of primary or revision ESS creating a management dilemma with their ASA use.

\section{Case report}

Recently a 19-year old Caucasian female patient presented with this scenario. This patient was having asthma symptoms of wheezing, shortness of breath, dyspnea, and coughing after ingesting ibuprofen. She was referred by her primary care physician to a medical allergist and after consultation and testing including in vivo testing for respiratory allergies which was negative was diagnosed with AERD by NSAID provocation challenge testing. Completing the aspirin desensitization protocol was extremely challenging and difficult for this patient requiring 3 attempts. However, once the aspirin desensitization protocol was completed the patient tolerated her aspirin desensitization maintenance therapy regimen of $650 \mathrm{mg}$ of aspirin twice daily very well.

Because the patient had symptoms of anosmia, facial pressure, nasal congestion and obstruction with clear and colored postnasal drainage the patient was referred to otolaryngology. Nasal endoscopy revealed severe bilateral nasal polyp disease and a severe right greater than left nasal septal deviation. A paranasal sinus CT scan was performed which showed severe pan-rhinosinusitis and nasal airway soft tissue densities consistent with the patient's nasal polyp disease found on nasal endoscopy (Figure 1,2). Endoscopic sinus surgery with nasal polypectomy, septoplasty and modified bilateral submucosal resection

*Correspondence to: Nishioka GJ, Willamette Ear Nose and Throat \& Facial Plastic Surgery, Salem, Oregon, USA, E-mail: garyn@entsalem.com

Key words: aspirin desensitization, aspirin exacerbated respiratory disease, endoscopic sinus surgery

Received: July 04, 2018; Accepted: July 20, 2018; Published: July 24, 2018 


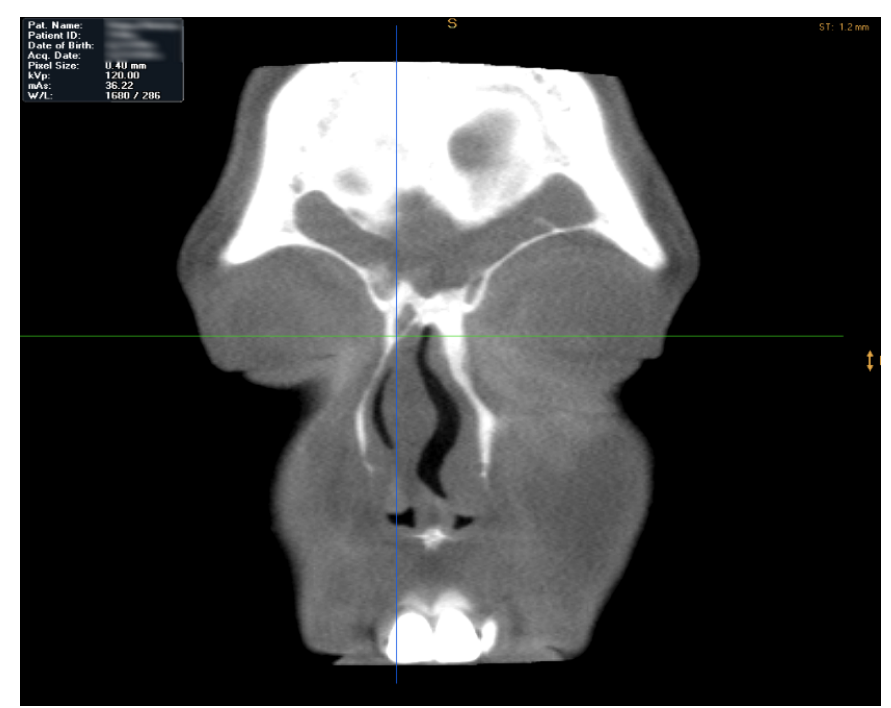

Figure 1. A preoperative paranasal sinus CT scan frame demonstrating radiographic findings of significant frontal sinus disease, and a right nasal septal deviation

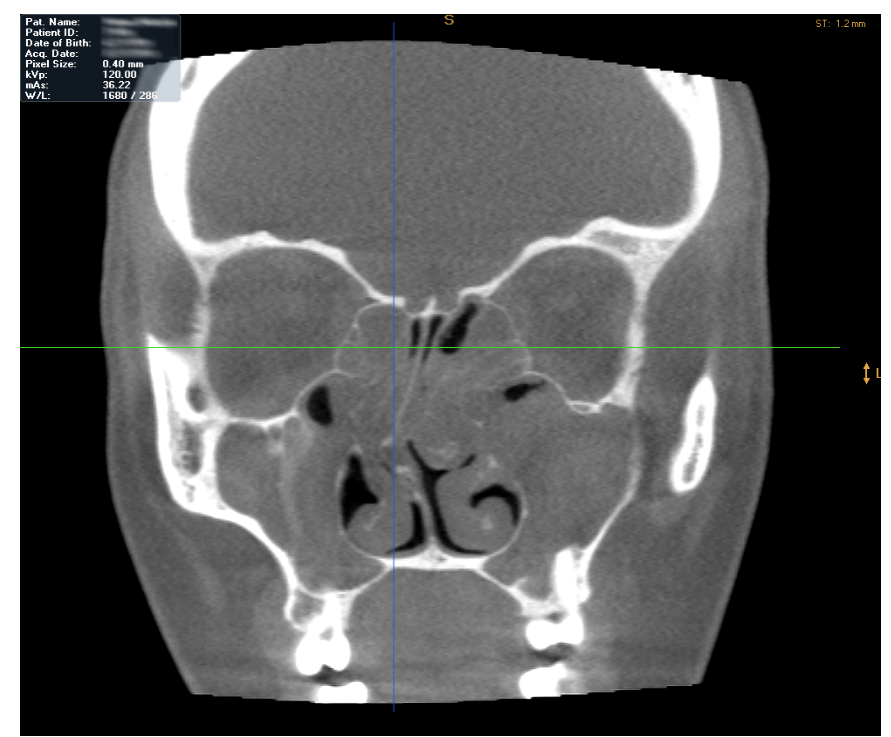

Figure 2. A preoperative paranasal sinus CT scan frame demonstrating radiographic findings of significant paranasal sinus and nasal polyp disease, and right nasal septal deviation

of the inferior turbinates was offered to the patient and was discussed in detail with the patient and parents.

After a comprehensive discussion with the patient and parents of whether to continue or stop her aspirin desensitization maintenance therapy it was mutually decided to proceed with ESS and continue aspirin desensitization maintenance therapy uninterrupted because of the significant morbidity of the ADT protocol itself and the concern that the patient would not be able to complete the ADT protocol again given her prior history.

The patient underwent extensive ESS of all the paranasal sinuses (including nasal polypectomy) with the aid of sinus CT navigation. Septoplasty and modified submucosal resection of the inferior turbinates were also performed. All planned procedures were easily performed with only a $100 \mathrm{ml}$ blood loss. No nasal packs were placed except for a resorbable dressing (Nasopore ${ }^{\mathrm{R}}$, Stryker Neuro Spine ENT) to each ethmoidectomy cavity site. Bleeding postoperatively was minimal, and recovery was routine.

\section{Discussion}

Triad asthma/AERD patients present with some of the most severe sinus disease with the majority if not all patients requiring ESS. The prevalence of ADT appears to be increasing and otolaryngologist will be encountering more patients on ADT maintenance therapy who will need primary or revision ESS creating a dilemma regarding stopping or continuing ASA uninterrupted. Stopping ASA prior to and for a period of time after ESS could require a patient to go through the ADT protocol again. If the ADT protocol was extremely difficult as it was for this patient, the prospect of enduring significant morbidity and possible failure of another ADT protocol resulted in the decision to proceed with ESS and continue ADT maintenance therapy uninterrupted. While stopping ASA or NSAIDs prior to ESS is a universal tenant there are situations such this that the surgeon may diverge successfully as was experienced in this case. Otolaryngologists who encounter similar situations such as this one can use this case report to help guide their clinical decision making regarding stopping or continuing aspirin desensitization maintenance therapy in AERD patients in need of ESS.

\section{References}

1. Lee RU, Stevenson DD (2011) Aspirin-exacerbated respiratory disease: evaluation and management. Allergy Asthma Immunol Res 3: 3-10. [Crossref]

2. Raja JP, Wineineger NE, Stevensen DD, White AA (2015) Prevalence of aspirin exacerbated respiratory disease among asthmatics: a meta-analysis of the literature. $J$ Allergy Clin Immunol 135: 676-681. [Crossref]

3. Adappa ND, Ranasinghe VJ, Trope M, Brooks SG, Glicksman JT, et al. (2018) Outcomes after complete endoscopic sinus surgery and aspirin desensitization in aspirin-exacerbated respiratory disease. Int Forum Allergy Rhinol 8: 49-53. [Crossref]

4. Cho KS, Soudry E, Psaltis AJ, Nadeau KC, McGhee SA, et al. (2014) Long-term sinonasal outcomes of aspirin desensitization in aspirin exacerbated respiratory disease. Otolaryngol Head Neck Surg 151: 575-581. [Crossref]

Copyright: (C2018 Nishioka GJ. This is an open-access article distributed under the terms of the Creative Commons Attribution License, which permits unrestricted use, distribution, and reproduction in any medium, provided the original author and source are credited. 